

\title{
Comparing C.E. Sets Based on Their Settling Times
}

\author{
Barbara F. Csima \\ Department of Pure Mathematics \\ University of Waterloo \\ Waterloo, ON, Canada N2L 3G1 \\ www.math.uwaterloo.ca/ ${ }^{\sim}$ csima \\ csima@math. uwaterloo.ca
}

\begin{abstract}
To each computable enumerable (c.e.) set $A$ with a particular enumeration $\left\{A_{s}\right\}_{s \in \omega}$, there is associated a settling function $m_{A}(x)$, where $m_{A}(x)$ is the last stage when a number less than or equal to $x$ was enumerated into $A$. In [7], R.W. Robinson classified the complexity of c.e. sets into two groups of complexity based on whether or not the settling function was dominant. An extension of this idea to a more refined ordering of c.e. sets was first introduced by Nabutovsky and Weinberger in [6] and Soare [9], for application to differential geometry. There they defined one c.e. set $A$ to settling time dominate another c.e. set $B$ $\left(B>_{\mathrm{st}} A\right)$ if for every computable function $f$, for all but finitely many $x, m_{B}(x)>f\left(m_{A}(x)\right)$. In [4] Csima and Soare introduced a stronger ordering, where $B>_{\text {sst }} A$ if for all computable $f$ and $g$, for almost all $x, m_{B}(x)>f\left(m_{A}(g(x))\right)$. We give a survey of the known results about these orderings, make some observations, and outline the open questions.
\end{abstract}

\section{Introduction}

An integral part of a computably enumerable (c.e.) set is it's enumeration. Obviously, a c.e. set has more than one enumeration, so when attempting to compare c.e. sets based on their settling times, it might be more correct to compare particular enumerations of the sets. It turns out that the notions introduced by Robinson, Nabutovsky, Weinberger and Soare, are all enumeration independent, so it is possible to compare c.e. sets, not just particular enumerations.

For Computability Theory, we follow the notation of Soare's Recursively Enumerable Sets and Degrees [8] and new notation from Soare's Computability Theory and Applications [10], which we also define. See also Cooper's Computability Theory [1] for a modern treatment of the subject.

We let $\left\{W_{e, s}\right\}_{e, s \in \omega}$ be any standard enumeration of the c.e. sets. We write " $\forall{ }^{\infty} x$ " for "for all but finitely many $x$ ".

We first recall the definition of a dominant function.

* Partially supported by Canadian NSERC Discovery Grant 312501. 
Definition 1. A function $g$ is said to be dominant if for every computable function $f,\left(\forall^{\infty} x\right)[g(x)>f(x)]$.

We also recall Martin's characterization of high sets in terms of dominant functions.

Theorem 1 (Martin [5]). A set $A$ is high $\left(A^{\prime} \equiv_{T} \emptyset^{\prime \prime}\right)$ if and only if there exists an A-computable dominant function.

We now give the definition of the settling function associated to an enumeration of a c.e. set.

Definition 2. For every computably enumerable (c.e.) set $W_{e}$ and associated enumeration $\left\{W_{e, s}\right\}_{s \in \omega}$, we define the settling (or modulus) function: $m_{e}(x)=$ $(\mu s)\left[W_{e, s} \Uparrow x=W_{e} \Uparrow x\right]$ where $A \Uparrow x=\{y \leq x \mid y \in A\}$.

In [7], Robinson sorted c.e. sets into two groups depending on whether or not their settling functions were dominant.

Definition 3. For every c.e. set $W_{e}$, we say that $W_{e}$ is dominant if its settling function is; that is if

$$
(\forall \text { computable } f)\left(\forall^{\infty} x\right)\left[m_{e}(x)>f(x)\right]
$$

Robinson referred to dominant sets as "high" and non-dominant sets as "low"; we will avoid this terminology due to the obvious confusion it would cause with the currently prevalent meaning of these words. Robinson showed that if a set has one enumeration that is dominant, then all are dominant, so the definition is independent of the enumeration chosen.

For use in an application to differential geometry, Nabutovsky and Weinberger [6] and Soare [9] introduced the following ordering on c.e. sets based on their settling times.

Definition 4. For c.e. sets $A$ and $B$, we say $A$ settling time dominates $B$ and write $A>_{s t} B$ iff $\left(\exists W_{i}=A\right)\left(\exists W_{j}=B\right)$

$$
(\forall \text { computable } f)\left(\forall^{\infty}\right)\left[m_{i}(x)>f\left(m_{j}(x)\right)\right] .
$$

Andre Nies showed that this is equivalent to $\left(\forall W_{i}=A\right)\left(\forall W_{j}=B\right)$ [(1) holds]. $W e$ denote the structure of the c.e. sets with the relation $<_{\mathrm{st}}$ as $\mathcal{E}_{\mathrm{st}}$.

Nabutovsky and Weinberger wanted a descending sequence in $\mathcal{E}_{\text {st }}$ to use in the construction of various manifolds that gave information on the geometry of Riemannian metrics modulo diffeomorphisms. Soare constructed such a sequence as described in [9]. See Soare [9], Csima and Soare [4], and Weinberger [11], for general background information on the ordering and its applications to differential geometry.

Though not designed for that purpose, the ordering $<_{\text {st }}$ gives a natural extension of the idea of domination to an ordering on c.e. sets. Indeed, we make the following observation. 
Observation 2. For a c.e. set A, the following are equivalent:

(i) $A$ is dominant.

(ii) There exists an infinite computable set $C$ such that $A>_{\mathrm{st}} C$.

(iii) $A>_{\mathrm{st}} C$ for every computable set $C$.

Proof. Clearly (iii) $\Rightarrow$ (ii).

For $(i) \Rightarrow(i i i)$, suppose $A$ is a dominant c.e. set, and that $C$ is any computable set. Then $m_{C}$ is computable, so for any computable function $f, f \circ m_{C}$ is computable. Thus since $A$ is dominant, $\left(\forall^{\infty} x\right)\left[m_{A}(x)>f\left(m_{C}(x)\right)\right]$, and so $A>{ }_{\text {st }} C$.

For (ii) $\Rightarrow(i)$, suppose that $A$ settling time dominates an infinite computable set $C$. Since $C$ is infinite and computable, then it has an enumeration such that $m_{C}$ is computable and such that $m_{C}(x) \geq x$ for almost every $x$. Let $f$ be any computable function. Let $f^{*}$ be a non-decreasing computable function such that $f^{*}(x) \geq f(x)$ for all $x$. Then since $A>_{\text {st }} C$, and since $f^{*}$ is computable, $\left(\forall^{\infty} x\right)\left[m_{A}(x) \geq f^{*}\left(m_{C}(x)\right)\right]$. Since $f^{*}$ is non-decreasing and since $\left(\forall^{\infty} x\right)\left[m_{C}(x) \geq x\right]$, we have $\left(\forall^{\infty} x\right)\left[m_{A}(x) \geq f^{*}\left(m_{C}(x)\right) \geq f^{*}(x) \geq f(x)\right]$. Since $f$ was arbitrary, this shows that $A$ is dominant.

Along similar lines, Csima and Soare observed the following.

Observation 3 (Csima, Soare [4]). If $A>_{\mathrm{st}} B$ and $B$ is infinite then $A$ is dominant.

Just as Turing reducibility refines the dichotomy of computable/non-computable to an order on the non-computable sets, $<_{\text {st }}$ gives an order on the dominant sets.

However, $<_{\text {st }}$ is not the only obvious choice for extending the idea of domination. In [4] and [3], the following strong settling time domination was introduced.

Definition 5. For c.e. sets $A$ and $B$ we say $A$ strongly settling time dominates $B, A>_{\text {sst }} B$, if for all computable functions $f$ and $g$, for almost every $x$,

$$
m_{A}(x)>f\left(m_{B}(g(x))\right) .
$$

The associated strict partial ordering on c.e. sets is denoted by $\mathcal{E}_{\mathrm{sst}}$.

The original motivation for this came as follows. To help simplify proofs in differential geometry, Nabutovsky and Weinberger asked whether there exits a sequence $\left\{A_{i}\right\}_{i \in \omega}$ of c.e. sets such that

$$
(\forall \text { computable } f)(\forall n)\left(\forall^{\infty} x\right)\left[m_{A_{i}}(x)>f\left(m_{A_{i+1}}(n x)\right)\right]
$$

This question was answered in my thesis [2] as follows.

Definition 6. Let $g$ be a computable function. For c.e. sets $A$ and $B$ we say $A>_{g-\text { st }} B$ if for all computable functions $f$, for almost every $x$,

$$
m_{A}(x)>f\left(m_{B}(g(x))\right) .
$$


Theorem 4. For any computable g, there exists a sequence $\left\{A_{i}\right\}$ of c.e. sets such that

$$
A_{i}>_{g-\mathrm{st}} A_{i+1}
$$

This answered the question of Nabutovsky and Weinberger using $g(x)=x^{2}$.

However, this also raised the question of whether there are sets $A$ and $B$ such that $A>_{g-\text { st }} B$ for all computable $g$. That is, whether there exist $A$ and $B$ such that $A>$ sst $B$.

Observation 5. For a c.e. set $A$, the following are equivalent:

(i) A is dominant.

(ii) There exists an infinite computable set $C$ such that $A>_{\mathrm{sst}} C$.

(iii) $A>_{\text {sst }} C$ for every computable set $C$.

Proof. Clearly (iii) $\Rightarrow$ (ii).

For (ii) $\Rightarrow(i)$, if $A>_{\text {sst }} C$ for some infinite computable set $C$, then $A>_{\text {st }} C$, so by Observation $2, A$ is dominant.

For $(i) \Rightarrow(i i i)$, suppose $A$ is a dominant c.e. set, and that $C$ is any computable set. Then $m_{C}$ is computable, so for any computable functions $f$ and $g, f \circ m_{C} \circ g$ is computable. Thus since $A$ is dominant, $\left(\forall^{\infty} x\right)\left[m_{A}(x)>f\left(m_{C}(g(x))\right)\right]$, and so $A>{ }_{\text {sst }} C$.

Thus, relative to computable sets, the orderings $<_{\text {st }}$ and $<_{\text {sst }}$ behave the same way. However, the two orderings $<_{\text {st }}$ and $<_{\text {sst }}$ are distinct on the dominant sets; that is, $<_{\text {st }}$ is a proper refinement of $<_{\text {sst }}$.

\section{Dominant Sets}

We now summarize Robinson's results on dominant c.e. sets.

Observation 6 (Robinson [7]). A dominant set must be high.

Indeed, a c.e. set $A$ is dominant if it's settling function $m_{A}$ dominates every computable function. By Martin's characterization [5], a set is high if and only if it can compute a dominant function. Since $A$ can certainly compute $m_{A}$, and since $m_{A}$ is dominant, $A$ is high.

Robinson further showed that every high c.e. degree contains a dominant set.

Theorem 7 (Robinson [7]). Every high c.e. Turing degree contains a dominant c.e. set.

Robinson showed this by again using Martin's characterization of high sets in terms of dominant functions.

On the other hand, a high c.e. set need not be dominant. Indeed, Robinson showed the following. 
Theorem 8 (Robinson [7]). Every c.e. degree contains a non-dominant set.

Corollary 1 (Robinson [7]). The dichotomy of the c.e. sets into dominant and non-dominant does not respect Turing reducibility.

On the other hand, Robinson showed that wtt-reducibility is respected.

Theorem 9 (Robinson [7]). If $A$ is dominant and $A<_{\text {wtt }} B$ then $B$ is dominant.

\section{$3 \quad$ Settling Time Reducibility}

As we saw earlier, if $A$ is a c.e. set that settling time dominates an infinite c.e. set, then $A$ must be dominant. So when comparing sets using the $<_{\text {st }}$ reducibility, all sets except those on the bottom will be dominant (and hence high).

The $<_{\text {st }}$ ordering works by comparing initial segments of the sets to one another, as we can see by the following theorems. We first introduce/recall some notation.

Definition 7. (i) $A$ set $A$ is bounded Turing reducible to a set $B \quad\left(A \leq_{\mathrm{bT}} B\right)$ if $A \leq_{T} B$ and there is a computable function $h(x)$ and a Turing reduction $A=\Phi_{e}^{B}$ with use function $u(x) \leq h(x)$. This is commonly written $A<_{\mathrm{wtt}} B$, but we introduce this notation with an eye towards part (ii) of the definition.

(ii) $A$ set $A$ is identity bounded Turing reducible to $B\left(A \leq_{\mathrm{ibT}} B\right)$ if $A \leq_{\mathrm{bT}} B$ with $h(x)=x$, namely $A=\Phi_{e}^{B}$ with use function $u(x) \leq x$ for all $x$.

Theorem 10 (Csima, Soare [4]). The $<_{\text {st }}$ ordering is well defined on ibTdegrees. Indeed, let $A, B$, and $C$ be c.e. sets with enumerations $\left\{A_{s}\right\}_{s \in \omega},\left\{B_{s}\right\}_{s \in \omega}$, and $\left\{C_{s}\right\}_{s \in \omega}$, respectively. If $A<_{\mathrm{st}} B$ and $B \leq_{\mathrm{ibT}} C$ then $A<_{\mathrm{st}} C$. If $A \leq_{\mathrm{ibT}} B$ and $B<_{\mathrm{st}} C$ then $A<_{\mathrm{st}} C$.

On the other hand, the $<_{\text {st }}$ ordering is not well-defined on bT-degrees (wttdegrees). Indeed, it does not even preserve computable isomorphism.

Theorem 11 (Csima, Shore [3]). The order $<_{\mathrm{st}}$ is not well defined on 1degrees. Indeed, there exist c.e. sets $A$ and $B$ such that $A \equiv_{1} B$ but $A>_{\text {st }} B$.

Certainly if $A>_{\mathrm{st}} B$ then $A \geq_{\mathrm{T}} B$. From the above, we see it is possible for $A$ and $B$ to have the same Turing degree. Csima and Soare showed that it is possible to have a sequence of c.e. sets descending strictly in both the Turing degrees and in $\mathcal{E}_{\text {st }}$.

Theorem 12 (Csima, Soare [4]). There exists a sequence $\left\{A_{n}\right\}_{n \in \omega}$ of c.e. sets such that $A_{n}>_{\mathrm{T}} A_{n+1}$ and $A_{n}>_{\mathrm{st}} A_{n+1}$ for all $n$.

When $<_{\text {st }}$ was first introduced, it was for the application to differential geometry, which only required a descending sequence as above. However, the natural question arose as to what kind of partial orders can be embedded into $\mathcal{E}_{\text {st }}$. 
Theorem 13 (Csima, Shore [3]). Any countable partial ordering can be embedded into $\mathcal{E}_{\text {st }}$.

Notice that $\mathcal{E}_{\text {st }}$ is a strict partial ordering. Indeed, for any c.e. set $A, A \ngtr_{\text {st }} A$. The largest equivalence relation on $\mathcal{E}_{\text {st }}$ (or any strict partial order) that respects the given ordering and gives a reflexive partial ordering as a quotient is given by $A \equiv_{\text {st }} B \Leftrightarrow\left\{C \mid C>_{\text {st }} A\right\}=\left\{C \mid C>_{\text {st }} B\right\} \&\left\{C \mid C<_{\text {st }} A\right\}=\left\{C \mid C<_{\text {st }} B\right\}$. One could instead of $\mathcal{E}_{\text {st }}$ then reasonably study its quotient $\mathcal{E}_{\text {st }}^{*}$ by this equivalence relation with the natural partial ordering $\leq$. Note that the above theorem shows that every countable partial ordering can be embedded in $\mathcal{E}_{\text {st }}^{*}$ as well, since a given partial order $P$ can be modified by adding on extra elements to produce a partial order $P^{\prime}$ such that any embedding of $P^{\prime}$ into $\mathcal{E}_{\text {st }}$ will restrict to one of $P$ into $\mathcal{E}_{\text {st }}^{*}$.

Theorem 14 (Csima, Shore [3]). There exists a maximal set in $\mathcal{E}_{\mathrm{st}}$. That is, there exists an $A$ such that for all $e, W_{e} \ngtr_{\text {st }} A$.

As for minimal sets, certainly any set that is not high would be minimal. If we consider the computable sets to be the simplest, then we can ask for the existence of a non-trivial minimal set in the sense that $A>_{\text {st }} C$ for any computable set $C$, but there exist no $B$ with $A>_{\text {st }} B>_{\text {st }} C$.

Theorem 15 (Csima, Shore [3]). There exists a non-trivial minimal set in $\mathcal{E}_{\mathrm{st}}$. Indeed, there exists a c.e. set $A$ such that $A>_{\mathrm{st}} C$ for every computable $C$, and if $W_{e}$ is noncomputable then $A \ngtr_{\mathrm{st}} W_{e}$.

Also, in [3], it is shown that infs and sups need not exist in $\mathcal{E}_{\text {st }}$.

Theorem 16 (Csima, Shore [3]). There are c.e. sets $A$ and $B$ such that $A$ and $B$ have no infimum in the $<_{\text {st }}$ ordering, indeed, such $A$ and $B$ can be found with $A \not \equiv_{\mathrm{st}} B$. There are c.e. sets $C$ and $D$ such that $C$ and $D$ have no supremum in the $<_{\mathrm{st}}$ ordering, indeed, such $C$ and $D$ can be found with $C \neq_{\mathrm{st}} D$.

The above theorem is proved by embedding a particular finite partial ordering into $\mathcal{E}_{\text {st }}$ with an added property that two of the sets have a gap between them. That is, the two sets $A$ and $C$ are such that $A>_{\text {st }} C$, but there is no c.e. set $H$ with $A>_{\text {st }} H>_{\text {st }} C$. The strategy for constructing two sets with a gap between them is similar to that of constructing a minimal set.

\section{Strong Settling Time Reducibility}

Clearly if $A>_{\text {sst }} B$, then $A>_{\text {st }} B$, so that $<_{\text {sst }}$ is a collapsing of $<_{\text {st }}$. Hence sets in this ordering are still all high (except for the ones on the bottom).

Unlike $<_{\text {st }}$, the ordering $<_{\text {sst }}$ is well defined on bT-degrees (wtt-degrees). This difference also shows that the two orderings are distinct.

Theorem 17 (Csima, Shore, [3]). The $<_{\text {sst }}$ ordering is well defined on $b T$ degrees. In fact, it respects bT-reducibility in the sense that if $A \leq_{\mathrm{bT}} B<_{\mathrm{sst}} C$ or $A<_{\mathrm{sst}} B \leq_{\mathrm{bT}} C$ then $A<_{\mathrm{sst}} C$. 
The proof of this is essentially the same as the proof that $<_{\text {st }}$ is well defined on ibT-degrees, but the extra strength of $<_{\text {sst }}$ allows arbitrary computable functions to be absorbed, rather than just the identity function.

On the other hand, $<_{\text {sst }}$ is not well defined on Turing degrees.

Theorem 18. The $<_{\text {sst }}$ ordering is not well defined on Turing degrees. Indeed, if $A>_{\mathrm{sst}} B$ and $B$ is infinite, then there exists a c.e. set $C \equiv_{\mathrm{T}} A$ such that $C \ngtr$ sst $B$.

Proof. In [4], this same theorem is shown with $<_{\text {st }}$ in place of $<_{\text {sst }}$. But, if $A \ngtr_{\text {st }}$ $B$, then certainly $A \ngtr_{\text {sst }} B$. It can also be seen as an immediate consequence of Corollary 1 and Observation 2.

In as far as embedding of partial orderings, Csima and Shore have the following partial result, which amounts to showing that certain linear orders can be embedded into $\mathcal{E}_{\text {sst }}$.

Theorem 19 (Csima, Shore [3]). Let $P$ be a computable partial ordering on $\mathbb{N}$ with no infinite ascending sequence. There exists a computable sequence $\left\{A_{n}\right\}_{n \in \mathbb{N}}$ of c.e. sets such that if $m<_{P} n$ then $A_{m}<_{\text {sst }} A_{n}$.

Since we have shown the existence of maximal and minimal sets for $\mathcal{E}_{\text {st }}$, we get the following for free in $\mathcal{E}_{\text {sst }}$.

Theorem 20. There exists a maximal set in $\mathcal{E}_{\text {sst }}$.

Proof. By Theorem 14, there exists a c.e. set $A$ such that for all $e, W_{e} \ngtr$ st $A$. But then certainly for all $e, W_{e} \ngtr$ sst $A$.

Theorem 21. There exists a non-trivial minimal set in $\mathcal{E}_{\text {sst }}$. That is, there exists a c.e. set $A$ such that $A>_{\mathrm{sst}} C$ for any computable $C$, but for all $W_{e}$, if $A>_{\text {sst }} W_{e}$ then $W_{e} \ngtr_{\text {sst }} C$.

Proof. By Theorem 15, there exists a c.e. set $A$ such that for all computable $C$, $A>_{\mathrm{st}} C$, and for all $e$, if $W_{e}$ is non-computable, then $A \ngtr_{\mathrm{st}} W_{e}$. Now if $A>_{\mathrm{st}} C$ for all computable $C$, then $A$ is dominant, and so $A>_{\text {sst }} C$ for all computable $C$, by Observations 2 and 5 . And if $A \ngtr_{\mathrm{st}} W_{e}$ for all $e$, then certainly $A \ngtr_{\mathrm{sst}} W_{e}$ for all $e$. So the non-trivial minimal set in $\mathcal{E}_{\text {st }}$ is still non-trivial and minimal in $\mathcal{E}_{\text {sst. }}$

\section{Questions}

It was shown that any countable partial order can be embedded into $\mathcal{E}_{\text {st }}$.

Question 1. Can this be done while simultaneously embedding into the Turing degrees?

In Theorem 19, Csima and Shore embed certain linear orders into $\mathcal{E}_{\text {sst }}$. 
Question 2. Can arbitrary countable partial orders be embedded into $\mathcal{E}_{\text {sst }}$ ?

The techniques of Theorem 19 can probably more quickly be adapted to answer the following easier question in the affirmative.

Question 3. Can every finite partial order be embedded into $\mathcal{E}_{\text {sst }}$ ?

We have seen that infs and sups need not exist in $\mathcal{E}_{\text {st }}$.

Question 4. Must infs and sups exist in $\mathcal{E}_{\text {sst }}$ ?

To prove that infs and sups do not exist in $\mathcal{E}_{\text {sst }}$, the argument for showing there is a minimal set was lifted to a more complicated setting. The way the existence of a non-trivial minimal set in $\mathcal{E}_{\text {sst }}$ was shown was to observe that the non-trivial minimal set in $\mathcal{E}_{\text {st }}$ was still non-trivial in $\mathcal{E}_{\text {sst }}$. This was because $<_{\text {st }}$ and $<_{\text {sst }}$ behave the same relative to the computable sets; though of course this is not true in general. So it is unclear whether the gap argument can go through in the $\mathcal{E}_{\text {sst }}$ setting. The following is probably true, and would be a good first step towards showing infs and sups do not exist in $\mathcal{E}_{\text {sst }}$.

Question 5. Do there exist non-computable $A$ and $B$ such that $A>_{\text {sst }} B$ and such that there exist no $C$ with $A>$ sst $C>$ sst $B$ ?

Certainly it cannot be true that given any high sets such that $A<_{\mathrm{T}} B$ then $A<_{\text {st }} B$, since Robinson already showed this must fail. However, Robinson did show that every high c.e. degree contains a dominant set, so the following may be possible:

Question 6. If $\mathbf{a}<\mathbf{b}$ are high c.e. degrees, must there exist c.e. sets $A \in \mathbf{a}$ and $B \in \mathbf{b}$ such that $A<_{\text {st }} B$ ? $A<_{\text {sst }} B$ ?

Question 7. More generally, given any partial order of high degrees, can there be realized a partial order of c.e. sets with the same relationship under $<_{\text {st }}\left(<_{\text {sst }}\right)$ in exactly those high degrees?

\section{References}

1. S. B. Cooper, Computability Theory, Chapman \& Hall/CRC, Boca Raton, FL, 2004.

2. B. F. Csima, Applications of Computability Theory to Prime Models and Differential Geometry, Ph.D. thesis, The University of Chicago, 2003.

3. B. F. Csima and R. A. Shore, The Settling-Time Reducibility Ordering, Journal of Symbolic Logic, $\mathbf{7 1}$ no. 4 (2006), 1394-1410.

4. B. F. Csima and R. I. Soare, Computability Results Used in Differential Geometry, Journal of Symbolic Logic, to appear.

5. D.A. Martin, Classes of recursively enumerable sets and degrees of unsolvability, Z. Math. Logik Grundlagen Math., 12 (1966), 295-310.

6. A. Nabutovsky and S. Weinberger, The Fractal Nature of Riem/Diff I, Geometrica Dedicata 101 (2003), 1-54. 
7. R. W. Robinson, A dichotomy of the recursively enumerable sets, Z. Math. Logik Grundlag. Math. 14 (1968), 339-356.

8. R. I. Soare, Recursively Enumerable Sets and Degrees: A Study of Computable Functions and Computably Generated Sets, Springer-Verlag, Heidelberg, 1987.

9. R. I. Soare, Computability theory and differential geometry, Bull. Symb. Logic 10 (2004), 457-486.

10. R. I. Soare, Computability Theory and Applications, Springer-Verlag, Heidelberg, to appear.

11. S. Weinberger, Computers, rigidity and moduli. The large scale fractal geometry of Reimannian moduli space, M.B. Porter Lectures, Princeton UNiversity Press, Princeton NJ, 2005. 